\title{
On Convergence a Variation of the Converse of Fabry Gap Theorem
}

\author{
Naser Abbasi, Molood Gorji* \\ Department of Mathematics, Lorestan University, Khoramabad, Islamic Republic of Iran
}

Email address:

naserAbbasi_persia@yahoo.com (N. Abbasi),molodgorji@yahoo.com (M. Gorji)

\section{To cite this article:}

Naser Abbasi, Molood Gorji. On Convergence a Variation of the Converse of Fabry Gap Theorem. Science Journal of Applied Mathematics and Statistics. Vol. 3, No. 2, 2015, pp. 58-62. doi: 10.11648/j.sjams.20150302.15

\begin{abstract}
In this article we give a variation of the converse of Fabry Gap theorem concerning the location of singularities of Taylor-Dirichlet series, on the boundary of convergence. whose circle of convergence is the unit circle and for which the unit circle is not the natural boundary.
\end{abstract}

Keywords: Dirichlet Series, Entire Functions, Fabry Gap Theorem

\section{Introduction}

The gap theorem of Fabry states that if $f(z)=\sum a_{k} e^{-\lambda z}$ is a power series whose circle of convergence is the unit circle and $\lim n_{k} / k=\infty$ then the unit circle is the natural boundary of $f(z)$.

Polya ([7]) proved the following converse of this result:

Let $n_{k}$ be a sequence of integers for which $\liminf n_{k} / k<\infty$; then there exists a power series $\sum a_{k} e^{-\lambda z}$ whose circle of convergence is the unit circle and for which the unit circle is not the natural boundary.

Based on a sequence $A$ as mentioned earlier, we construct a multiplicity sequence $B=\left\{\left(\lambda_{n}, \mu_{n}\right)\right\}_{n=1}^{\infty}$, that is, a sequence where, for $n \neq m$ one has $\lambda_{n} \neq \lambda_{m}, \quad\left|\lambda_{n}\right| \leq\left|\lambda_{n+1}\right|$ and each $\lambda_{n}$ appears $\mu_{n}$ times. For this sequence $B$ we prove that the infinite product $G(z)$ which vanishes exactly on $\pm B$, satisfies $\mu_{n} ! /\left|G^{\left[\mu_{n}\right]}\left(\lambda_{n}\right)\right|=O\left(\exp \left\{\epsilon\left|\lambda_{n}\right|\right\}\right)$ every $\epsilon>0$. That is, we have a sharp estimate for the $\mu_{n}$ th derivative function of $G(z)$ evaluated on $\lambda_{n}$.

We assume that the reader is familiar with the theory of Entire Functions and the theory of Dirichlet series, as used in the books [1,5,8-11].

We note that other results concerning the location of singularities of Taylor-Dirichlet series have been derived by Blambert, Parvatham, and Berland (see [2-4]).

\section{Auxiliary Results and Notions}

In this section, we describe the definitions and also to express and prove the lemma, we need to prove the theorem.

Definition 2.1. We denote by $L(c, D)$ the class of all sequences $A=\left\{a_{n}\right\}_{n=1}^{\infty}$ with distinct complex terms $a_{n}$ diverging to infinity, $\left|a_{n}\right| \leq\left|a_{n+1}\right|$ satisfying the following conditions: (see also [12])

(1) There is a constant $c>0$ so that $\left|a_{n}-a_{m}\right| \geq c|n-m|$ for all $n \neq m$.

(2) $\lim _{n \rightarrow \infty} n /\left|a_{n}\right|=D \geq 0$.

(3) the $\sup \left|\arg a_{n}\right|<\pi / 2$.

Definition 2.2. Choose a sequence $A=\left\{a_{n}\right\}_{n=1}^{\infty}$ which belongs to the class $L(c, D)$. suppose $\alpha$ and $\beta$ be real positive numbers so that $\alpha+\beta<1$. We say that a sequence $B=\left\{b_{n}\right\}_{n=1}^{\infty}$ with complex terms $b_{n}, b_{n} \neq 0$, with the $\left|b_{n}\right|$ not necessarily in an increasing order, and $\sup _{n \in N}\left|\arg b_{n}\right| \leq \pi / 2$, belongs to the class $A_{\alpha, \beta}$ if for all $n \in N$ we have $b_{n} \in\left\{z \in C:\left|z-a_{n}\right| \leq\left|a_{n}\right|^{\alpha}\right\}$, and for all $m \neq n$ one of the following conditions holds:

(i) $b_{m}=b_{n}$

(ii) $\left|b_{m}-b_{n}\right| \geq \max \left\{e^{-\left|a_{m}\right|^{\beta}}, e^{-\left|a_{n}\right|^{\beta}}\right\}$.

One observes that $(i)$ allows for the sequence $B$ to have coinciding terms. We may now rewrite $B$ in the form of a multiplicity sequence $\left\{\lambda_{n}, \mu_{n}\right\}_{n=1}^{\infty}$ in the following way; first 
we split $B$ into groups of terms having the same modulus, and then within each group we order them by the size of their argument, beginning from smaller to larger. The arguments are taken in the interval $0 \leq \arg b_{n} \leq 2 \pi$. We shall say that $\left\{\lambda_{n}, \mu_{n}\right\}$ is the $(\lambda, \mu)$ reordering of $B$.

Remark. We point out that the spacing condition (2) of a sequence $A \in L(c, D)$ plays a very important role throughout the article.

Let $\Gamma_{n}$ be as in

$$
\Gamma_{n}=\left\{j: b_{j}=b_{n}\right\} .
$$

One deduces that if $j \in \Gamma_{n}$ then $\Gamma_{j}=\Gamma_{n}$. We also define $m(n)$ to be the number of terms of $\Gamma_{n}$ and we shall refer to $m(n)$ as the pseudo-multiplicity of $b_{n}$.

In the lemma that follows, we get an upper bound for $m(n)$ with respect to $b_{n}$.

Lemma 2.3. There exist positive constants $\psi$ and $\chi$ so that for any $n$ one has

$$
m(n) \leq \psi\left|a_{n}\right|^{\alpha} \leq \chi\left|b_{n}\right|^{\alpha}
$$

Proof. First note that the relation

$$
\left|a_{n}\right| / 2 \leq\left|b_{n}\right| \leq 2\left|a_{n}\right|
$$

holds for all $n>n_{0}$ since $\left|a_{n}-b_{n}\right| \leq\left|a_{n}\right|^{\alpha}$. Consider now any $j \in \Gamma_{n}\left(\Gamma_{n}=\left\{j: b_{j}=b_{n}\right\}\right)$. Then

$$
\begin{aligned}
\left|a_{j}\right|=\mid\left(a_{j}-b_{j}\right)+ & \left(b_{j}-b_{n}\right)+\left.b_{n}|\leq| a_{j}\right|^{\alpha}+\left|b_{n}\right| \\
& \leq \frac{\left|a_{j}\right|}{2}+2\left|a_{n}\right|
\end{aligned}
$$

It follows that $\left|a_{j}\right| \leq 4\left|a_{n}\right|$. Then one also gets

$$
\begin{gathered}
\left|a_{n}-a_{j}\right|=\left|\left(a_{n}-b_{n}\right)+\left(b_{n}-b_{j}\right)+\left(b_{j}-a_{j}\right)\right| \\
\leq\left|a_{n}\right|^{\alpha}+\left|a_{j}\right|^{\alpha} \leq 5\left|a_{n}\right|^{\alpha}
\end{gathered}
$$

Finally, the spacing condition

$$
\left|a_{n}-a_{k}\right| \geq c|n-k|
$$

yields that for any $j \in \Gamma_{n}$ one has

$$
c|j-n| \leq 5\left|a_{n}\right|^{\alpha}
$$

Since $m(n)$ is the number of terms of $\Gamma_{n}$, then $m(n) \leq 2 \max \left\{|j-n|: j \in \Gamma_{n}\right\}$. From the above equation it follows that there exists a positive $\psi$ so that $m(n) \leq \psi\left|a_{n}\right|^{\alpha}$. Finally, the relation $\left|a_{n}\right| \leq 2\left|b_{n}\right|$ yields a positive $\chi$ so that $m(n) \leq \chi\left|b_{n}\right|^{\alpha}$.

Lemma 2.4. For any $n$ one has

$$
\mu_{n} \leq \chi\left|\lambda_{n}\right|^{\alpha}
$$

Proof. Let $\lambda_{n}=b_{k}$ for some $k \in N^{+}$. From the previous lemma we know that $m(k) \leq \chi\left|b_{k}\right|^{\alpha}$ for some $\chi>0$. But the pseudo-multiplicity $m(k)$ of $b_{k}$ is the multiplicity $\mu_{n}$ of $\lambda_{n}$. Thus, one obtains the relation $\mu_{n} \leq \chi\left|\lambda_{n}\right|^{\alpha}$.

Another important lemma, which is important for this paper can be stated as follows:

Lemma 2.5.Let $A \in L(c, D)$ be a real positive sequence and let $B \in A_{\alpha, \beta}$ so that $B=\left\{b_{n}\right\}$ is real positive too, with $(\lambda, \mu)$ its reordering. Then the regions of convergence of the three series $f, f^{*}, f^{* *}$ as defined in

$$
f(z)=\sum_{n=1}^{\infty} p_{\mu_{n}}(z) e^{-\lambda_{n} z}
$$

where $p_{\mu_{n}}(z)=\sum_{j=0}^{\mu_{n}-1} c_{n_{j}} z^{j}$ is a polynomial with $c_{n_{\mu_{n}-1}} \neq 0$, and

$$
f^{*}(z)=\sum_{n=1}^{\infty} A_{n} e^{-\lambda_{n} z}, \quad f^{* *}(z)=\sum_{n=1}^{\infty} A_{n} z^{\mu_{n}-1} e^{-\lambda_{n} z}
$$

are the same. For any point $z$ inside the open convex region, the three series converge absolutely. Similarly, if instead of a real sequence $A \in L(c, D)$ we have a complex sequence $A \in L(c, 0)$.

Proof. We have to show that

$$
\lim _{n \rightarrow \infty} \frac{\log n}{\lambda_{n}}=0, \quad \lim _{n \rightarrow \infty} \frac{\mu_{n}-1}{\lambda_{n}}=0,
$$

is satisfied. First, note that from Lemma 2.4 one deduces that the right limit of

$$
\lim _{n \rightarrow \infty} \frac{\log n}{\lambda_{n}}=0, \quad \lim _{n \rightarrow \infty} \frac{\mu_{n}-1}{\lambda_{n}}=0,
$$

is valid. Thus, it remains to verify the left limit.

We claim that

$$
\left|\lambda_{n}\right| \geq\left|a_{n}\right| / 2, \quad n \geq 1
$$

This implies that

$$
\frac{\log n}{\left|\lambda_{n}\right|}=\frac{\log n}{n} \frac{n}{\left|a_{n}\right|} \frac{\left|a_{n}\right|}{\left|\lambda_{n}\right|} \leq \frac{\log n}{n} \frac{n}{\left|a_{n}\right|} \frac{1}{2} \rightarrow 0, \quad n \rightarrow \infty
$$

since $n /\left|a_{n}\right| \rightarrow D$, and we are done.

Let us justify our claim. It is obvious that $\left|\lambda_{1}\right| \geq\left|a_{1}\right| / 2$. Assume that $\left|\lambda_{k}\right| \geq\left|a_{k}\right| / 2$ for some $k$. We will prove that 
$\left|\lambda_{k+1}\right| \geq\left|a_{k+1}\right| / 2$ as well.

Note that there is at least one $b_{n}$ so that $\lambda_{k+1}=b_{n}$. If $\left|\lambda_{k+1}\right|<\left|a_{k+1}\right| / 2$ then $\left|a_{n}\right| / 2 \leq\left|b_{n}\right|=\left|\lambda_{k+1}\right|<\left|a_{k+1}\right| / 2$.

Since $\left|a_{n}\right| / 2<\left|a_{k+1}\right|$, this implies that $n \leq k$ since $\left|a_{n}\right| \leq\left|a_{n+1}\right|$ for all $n \in \mathrm{N}$, therefore $\lambda_{k+1} \in\left\{b_{m}\right\}_{m=1}^{k}$. This means that $\left\{\left(\lambda_{m}, \mu_{m}\right)\right\}_{m=1}^{k} \neq\left\{b_{m}\right\}_{m=1}^{k}$, thus there is some $b_{j} \in\left\{\left(\lambda_{m}, \mu_{m}\right)\right\}_{m=1}^{k}$ with $j \geq k+1$. It follows that

$$
\frac{\left|a_{k+1}\right|}{2}>\left|\lambda_{k+1}\right| \geq\left|\lambda_{k}\right| \geq\left|b_{j}\right| \geq \frac{\left|a_{j}\right|}{2} \geq \frac{\left|a_{k+1}\right|}{2}
$$

that is, $\left|a_{k+1}\right|>\left|a_{k+1}\right|$ which is false. Thus $\left|\lambda_{k+1}\right| \geq\left|a_{k+1}\right| / 2$ and this completes the proof.

\section{Main Results}

This section describes the main theorem of this paper, and it can be fixed by using proven methods Polya.

Theorem 3.1. suppose $A \in L(c, D)$ be a real positive sequence. Let $B \in A_{\alpha, \beta}$ so that $B=\left\{b_{n}\right\}$ is real positive too $\liminf n \lambda_{n}^{-1}=D<\infty$ and let $(\lambda, \mu)$ be its reordering. Then any Taylor-Dirichlet series $f(z)$ as in (1), satisfying

$$
\limsup _{n \rightarrow \infty} \frac{\ln \left|c_{n_{\mu_{n}-1}}\right|}{\lambda_{n}}=\limsup \sup _{n \rightarrow \infty} \frac{\ln A_{n}}{\lambda_{n}}
$$

whose circle of convergence is the unit circle and for which the unit circle is not the natural boundary. [6].

Proof. We follow on the lines of the proof of Theorem in

Let $f, f^{*}, f^{* *}$ and $A_{n}$ as defined in (1), (2) and

$$
A_{n}=\max \left\{\left|c_{n_{j}}\right|: j=0,1,2, \ldots, \mu_{n}-1\right\} .
$$

From Lemma 2.5, the regions of convergence of the three series are the same. Since the $\lambda_{n}$ are real positive numbers, we consider the non-trivial case, that is when the three series converge in identical half-planes of the form $\Re z>x_{0}, x_{0} \in R$. With no loss of generality we assume that the abscissa of convergence (ordinary and absolute) is the line $x=1$.

In other words the relation

$$
\limsup \operatorname{sun}_{n \rightarrow \infty} \frac{\ln A_{n}}{\lambda_{n}}=1
$$

holds. Thus, all three series converge absolutely and uniformly in any half-plane $x \geq \tau>1$. One also notes that from (3) we have

$$
\limsup \operatorname{sun}_{n \rightarrow \infty} \frac{\ln \left|c_{n_{\mu_{n-1}}}\right|}{\lambda_{n}}=1
$$

There clearly exist two sequences of integers $B_{i}$ and $B_{j}$ such that $B_{i}=\left[\left(1+c_{1}\right) B_{j}\right], B_{j+1}>B_{i}^{2}$, and the number of $\lambda_{n}$ in $\left(B_{j}, B_{i}\right)$ is greater than $c_{2}\left(B_{i}-B_{j}\right)>c_{3} B_{j}$. (The c's denote absolute positive constants.) The existence of these sequences is immediate from $\liminf n / \lambda_{n}<\infty$. Denote the $\lambda_{n}$ in the intervals $\left(B_{j}, B_{i}\right)$ by $\lambda_{n^{\prime}}$. We clearly have $\liminf n / \lambda_{n}^{\prime}<\infty$. For construction of $f(z)$ we shall use only the $\lambda_{n^{\prime}}$. Put $f(z)=\sum_{n=1}^{\infty} p_{\mu_{n}}(z) e^{-\lambda_{n^{\prime} z}}$ We shall determine the $p_{\mu_{n}}$ so that the unit circle will be the circle of convergence and the point 1 will be a regular point of $f(z)$. It will suffice to show that there exists a number $1,1>l>0$, such that the circle of convergence of

$$
f(z+l)=\sum_{n} p_{\mu_{n}}(z+l) e^{-\lambda_{n}^{\prime}(z+l)}=\sum_{m} b_{m} e^{-m}
$$

has radius greater than $1-l$. We shall choose $l=(r-1) / r, r$ a sufficiently large integer. We have by the binomial expansion

$$
\sum_{n} p_{\mu_{n}}\left(z+\frac{r-1}{r}\right) e^{-\lambda_{n}^{\prime}\left(z+\frac{r-1}{r}\right)}=\sum_{m} e^{-m} \sum_{n} p_{\mu_{n}}\left(z+\frac{r-1}{r}\right) C_{\lambda_{n}^{\prime}, m}\left(\frac{r-1}{r}\right)^{\lambda_{n}^{\prime}-m}=\sum_{m} b_{m} e^{-m}
$$

We have to show that $\lim \sup b_{m}^{1 / m}<r$, for some choice of the $p_{\mu_{n}}$ with $\limsup \left|p_{\mu_{n}}\right|^{1 / \lambda_{n}}=1$.

Let $\epsilon$ be a small but fixed number; we distinguish two cases.

In case $(i), m$ does not lie in any of the intervals $\left(\left(B_{j} / r\right)(1-\epsilon),\left(B_{i} / r\right)(1+\epsilon)\right)$. Then we show that for every choice of the $p_{\mu_{n}}$ with $\left|p_{\mu_{n}}\right| \leq 1, \quad \limsup b_{m}^{1 / m}<r-\delta$, $\delta=\delta(\epsilon)$.

This means that if $m$ is large enough and does not lie in $\left(\left(B_{j} / r\right)(1-\epsilon),\left(B_{i} / r\right)(1+\epsilon)\right)$ then $b_{m}<(r-\delta)^{m}$. Clearly

$$
b_{m} \leq \sum_{n} C_{\lambda_{n}^{\prime}, m}\left(\frac{r-1}{r}\right)^{\lambda_{n}^{\prime}-m} .
$$

If we define

$$
C_{k, m}((r-1) / r)^{k-m}=H_{k}
$$

We find

$$
H_{k+1} / H_{k}=((r-1) / r)(k+1) /(k-m+1) .
$$

By studying the quotient (8) we see that $\max H_{k}=H_{r m}=C_{r m, m}((r-1) / r)^{(r-1) m}$ and by applying 
Stirling's formula

$$
k ! \sim(2 \pi)^{-1 / 2} k^{k+1 / 2} c^{-k}
$$

We note that $H_{r m}^{1 / m} \rightarrow r$ as $m \rightarrow \infty$. It follows from (8) that there exists $k=k(\epsilon)>0$ such that

$$
\begin{array}{lll}
H_{k+1} / H_{k}>1+\eta & \text { for } & n<r m /(1+\epsilon) \\
H_{k+1} / H_{k}<1-\eta & \text { for } & n>r m /(1-\epsilon)
\end{array}
$$

and hence a simple calculation shows that there exists a $\beta=\beta(\epsilon)>0$ such that

$$
H_{k}<(r-\beta)^{m}
$$

for $k$ not in $(\mathrm{rm} /(1+\epsilon), \mathrm{rm} /(1-\epsilon))$.

Now clearly

$$
b_{m}=\sum_{1}+\sum_{2}
$$

where in $\sum_{1}$ the summation is extended over the $k<r m /(1+\epsilon)$ and in $\sum_{2}$ over the $k>r m /(1-\epsilon)$. (By assumption $m$ does not lie in $\left(\left(B_{j} / r\right)(1-\epsilon),\left(B_{i} / r\right)(1+\epsilon)\right)$ and in (7) the $\lambda_{n}^{\prime}$ are all in $\left(B_{j}, B_{i}\right)$; thus if $m<\left(B_{j} / r\right)(1-\epsilon), \lambda_{n}^{\prime}>r m /(1-\epsilon)$ and if $m>\left(B_{i} / r\right)(1+\epsilon), \lambda_{n}^{\prime}<r m /(1+\epsilon)$.) Thus from (9), (10) and (11) (by summing a geometric series)

$$
b_{m}<c_{4}(r-\beta)^{m}
$$

or

$$
\lim \sup b_{m}^{1 / m}<(r-\delta)
$$

which completes the proof.

In case $(i i)$

$$
\left(B_{j} / r\right)(1-\epsilon)<m<\left(B_{i} / r\right)(1+\epsilon) .
$$

We write

$$
b_{m}=b_{m}^{\prime}+b_{m}^{\prime \prime},
$$

where

$$
\begin{aligned}
& b_{m}^{\prime}=\sum_{1} p_{\mu_{n}}\left(z+\frac{r-1}{r}\right) C_{\lambda_{n}^{\prime}, m}\left(\frac{r-1}{r}\right)^{\lambda_{n}^{\prime}-m}, \\
& b_{m}^{\prime \prime}=\sum_{2} p_{\mu_{n}}\left(z+\frac{r-1}{r}\right) C_{\lambda_{n}^{\prime}, m}\left(\frac{r-1}{r}\right)^{\lambda_{n}^{\prime}-m},
\end{aligned}
$$

$\sum_{1}$ indicates that the summation is extended only over those $n$ for which $\lambda_{n}^{\prime}$ does not lie in $\left(B_{j}, B_{i}\right)$, and in $\sum_{2}$ the summation is extended over the other $n$. We

$$
b_{m}^{\prime} \leq \sum_{1} C_{\lambda_{n}^{\prime}, m}\left(\frac{r-1}{r}\right)^{\lambda_{n}^{\prime}-m}
$$

and we can show that $\lim \sup b_{m}^{1 / m}<r$ as before.

Now we show that we can choose the $p_{\mu_{n}}$ to be such as to make all the $b_{m}^{\prime \prime}$ for $\left(B_{j} / r\right)(1-\epsilon) \leq m \leq\left(B_{i} / r\right)(1+\epsilon)$ equal to 0 . Thus we must determine the $p_{\mu_{n}}$ so that

$$
\sum_{2} p_{\mu_{n}}\left(z+\frac{r-1}{r}\right) C_{\lambda_{n}^{\prime}, m}\left(\frac{r-1}{r}\right)^{\lambda_{n}^{\prime}-m}=0
$$

These are homogeneous equations for the $p_{\mu_{n}}$. The number of these equations is less than $2\left(B_{j}-B_{i}\right) / r$ for sufficiently small $\epsilon$ and the number of unknowns is greater than $c_{3}\left(B_{j}-B_{i}\right)$ which is greater than the number of equations for large enough $r$. Thus the system of equations always has a solution, and further we can suppose that the absolute value of the largest $p_{\mu_{n}}$ is 1 . This will insure that the circle of convergence of $f(z)$ will be the unit circle, which completes the proof.

\section{Conclusion}

In this study we examine a variation of the converse of Fabry Gap theorem.

Polya's result shows that in some sense Fabry's result is the best possible. Perhaps the elementary and direct proof that mentioned above might be of some interest.

To do this, a sequence with a series of new build and reordering the call, using the convergence of three series $f, f^{*}, f^{* *}$ obtain upper and lower bounds. And using the Stirling's formula and we will achieve the desired result in this paper.

\section{Acknowledgements}

The author gratefully acknowledges the help of Prof. E. Zikkos to improve the original version of the paper.

\section{References}

[1] Berenstein, C.A., and Gay Roger, Complex Analysis and Special Topics in Harmonic Analysis (New York, Inc: Springer-Verlag), (1995).

[2] Blambert, M. and Parvatham, R., "Ultraconvergence et singualarites pour une classe de series d exponentielles." Universite de Grenoble. Annales de l'Institut Fourier, 29(1), (1979), 239-262.

[3] Blambert, M. and Parvatham, R., "Sur une inegalite 
fondamentale et les singualarites $\mathrm{d}$ une fonction analytique definie par un element LC-dirichletien. "Universite de Grenoble. Annales de l'Institut Fourier, 33(4), (1983), 135-160.

[4] Berland, M., "On the convergenve and singularities of analytic functions defined by E-Dirichletian elements. "Annales des Sciences Mathematiques du Quebec, 22(1),(1998), 1-15.

[5] Boas, R.P. Jr, , "Entire Functions," (New York: Academic Press), (1954).

[6] Erdos, P., "Note on the converse of Fabry's Gap theorem," Trans. Amer. Math. Soc., 57, (1945), 102-104.

[7] Polya, G., "On converse Gap theorems," Trans. Amer. Math. Soc., 52, (1942), 65-71.
[8] Levin, B. Ya., "Distribution of Zeros of Entire Functions," (Providence, R.I.: Amer. Math. Soc.), (1964).

[9] Levin, B. Ya., "Lectures on Entire Functions, " (Providence, R.I.: Amer. Math. Soc.), (1996).

[10] Levinson, N., "Gap and Density Theorems. "American Mathematical Society Colloquium Publications, Vol. 26 (New York: Amer. Math. Soc.), (1940).

[11] Mandelbrojt, S., "Dirichlet Series, Principles and Methods," (Dordrecht: D. Reidel Publishing Co.), (1972), pp. x166.

[12] Zikkos, E., "On a theorem of Norman Levinson and a variation of the Fabry Gap theorem," Complex Variables, 50 (4), (2005), 229-255. 